\title{
marges Marges
}

revue d'art contemporain Revue d'art contemporain

22 | 2016

L'Artiste-théoricien

\section{Éditorial}

Jérôme Glicenstein

\section{(2) OpenEdition}

Journals

Édition électronique

URL : http://journals.openedition.org/marges/1062

DOI : 10.4000/marges.1062

ISSN : 2416-8742

\section{Éditeur}

Presses universitaires de Vincennes

\section{Édition imprimée}

Date de publication : 22 avril 2016

Pagination : 5-7

ISBN : 978-2-84292-529-1

ISSN : 1767-7114

Référence électronique

Jérôme Glicenstein, «Éditorial », Marges [En ligne], 22 | 2016, mis en ligne le 22 avril 2016, consulté le 24 septembre 2020. URL : http://journals.openedition.org/marges/1062 ; DOI : https://doi.org/ $10.4000 /$ marges. 1062 


\section{Éditorial}

Selon un schéma hérité de la Renaissance - en un lointain souvenir de Léonard de Vinci ou de Leon Battista Alberti - les artistes se font à l'occasion théoriciens de leur propre pratique. Leurs écrits revêtent alors des formes très variées : essais, critiques, mémoires, notes, carnets, romans... suivant un modèle qui a été repris et accentué à l'époque des avant-gardes historiques. Dans certains cas, l'artiste en vient même à élaborer des programmes théoriques qui ne se limitent pas au champ de l'art (de Seurat au Futurisme, en passant par le Bauhaus ou le constructivisme). Plus près de nous, au milieu des années 1960, le modèle de l'artiste comme théoricien est repensé: l'art conceptuel, en établissant une équivalence entre art et discours sur l'art, donne alors pour objectif à la création de se définir par elle-même et des artistes comme Daniel Buren déclarent que la théorie est indissociable de leur pratique. Ces artistes entendent alors revendiquer une position de résistance face à l'hégémonie des discours institutionnels, tout en s'opposant aux catégorisations qu'ils imposent. Depuis la fin des années 1970, ce modèle semble être en perte de vitesse. En revanche, d'autres figures d'artistes apparaissent qui empruntent des éléments théoriques et méthodologiques à une grande diversité de champs disciplinaires: artiste-ethnographe, artiste-archiviste ou documentaliste, artiste-producteur, artiste-narrateur, artiste-cartographe, artiste-scientifique... Dans ces nouvelles postures, l'artiste s'intéresse davantage à des connaissances appliquées qu'à des élaborations spéculatives et laisse de côté l'idée d'une maîtrise complète de savoirs abstraits au profit de connaissances plus pratiques. Cette nouvelle situation suscite des questions. La théorie, produite ou importée par les artistes, 
soutient-elle nécessairement leur pratique? Quelle est la nature de cette théorie, quelles formes prend-elle et comment en juger? L'objet de ce numéro est d'interroger les différentes facettes et enjeux que recouvre l'activité théoricienne pour un artiste, les conséquences sur sa pratique ou sur son statut d'artiste.

Le premier texte conserve une certaine ambiguïté, ne serait-ce que parce que l'artiste dont il est question a eu d'autres activités que strictement artistiques. Gabriel Ferreira Zacarias revient ainsi sur le paradoxe apparent qui tient souvent Guy Debord pour un théoricien, alors que l'ensemble de son œuvre n'a eu de cesse de fonder un art "basé sur l'expérience ", qui ne se contenterait pas d'illustrer passivement des théories esthétiques. Face à ce genre de position, c'est un peu un mouvement inverse qui conduit Marcel Broodthaers et Joseph Kosuth à s'inspirer du Tractatus logico-philosophicus de Ludwig Wittgenstein. Pour ces deux artistes, ainsi que le montre Denis Laoureux, la référence très marquée à un texte théorique permet de s'interroger sur l'autonomie de l'expérience - même si ce texte n'est pas lu ou illustré littéralement.

L'idée d'un rapport détaché à l'élaboration théorique se retrouve dans le texte de Nina Leger qui s'intéresse, elle, aux élaborations théoriques de Robert Smithson et Mel Bochner, des artistes emblématiques du mouvement de théorisation des pratiques artistiques aux États-Unis dans les années 1960. Ces artistes, explique-t-elle, entretiennent une relation très singulière à la théorie, s'en servant plus comme d'un moyen (un outil parmi d'autres) que comme d'une fin en soi.

Nicolas Heimendinger s'interroge quant à lui sur le bien fondé d'une dénomination telle que «critique institutionnelle »- laquelle a souvent été utilisée pour qualifier les pratiques d'artistes des années 1960-70 - et sur la manière dont cette notion a été construite rétrospectivement par des artistes et théoriciens des années 1980-90, dans le but sans doute de redonner vigueur à des mouvances en perte de vitesse. Ici, les artistes ne sont pas seuls: ils font partie d'un ensemble plus large et sont pris pour objets de débats théoriques qui ne les concernent pas nécessairement. La pratique des artistes de ces dernières années conduit de fait à reconsidérer la notion d'artiste-théoricien. 
De nouvelles formes, telles que la conférence-performance, engagent en effet à inclure la dimension théorique d'une œuvre dans sa réalisation pratique. Les danseurs dont parle Giuseppe Burighel - Xavier Le Roy, Jérôme Bel, Claudia Triozzi - entretiennent ainsi avec leur pratique une relation qui n'est pas purement de l'ordre de l'élaboration théorique, même si elle joue d'une prise de distance métadiscursive, ce dont témoigne par exemple l'usage de narrations autobiographiques dans les conférences-spectacles.

Les deux textes suivants s'interrogent quant à eux sur l'importance accordée à la constitution d'un discours théorique lors de la formation des artistes. Cette question est en effet un enjeu de la normalisation des écoles d'art, depuis l'entrée en vigueur du processus de Bologne (1999). Jérémie Vandenbunder traite de cette question sous un angle sociologique - à travers une enquête menée au sein des écoles d'art alors qu'Evangelos Athanassopoulos s'interroge quant à lui sur ses implications multiples, avec le passage de la figure de l'artiste-théoricien à celle de l'artiste-chercheur au service de la production de nouvelles formes de valeur.

Dans les varias de ce numéro nous publions un article de Geneviève Chevalier sur les origines des cartes blanches proposées aux artistes afin de réaccrocher les collections permanentes de certains musées. L'auteure s'appuie en particulier sur le cas d'Andy Warhol et de son exposition « Raid the Icebox 1 » (1969-1970). Nous publions également un entretien mené par Sarah Heussaff avec Kamil Guenatri (La Terrasse), un artiste performeur dont la condition physique lui impose d'être constamment assisté - ce qui crée une relation ambivalente avec ses assistants de vie, également vecteurs de réalisation des performances.

Selon une tradition désormais bien ancrée, nous présentons deux interventions d'artiste: un portfolio de Noémie Goudal, Les Observatoires et une proposition textuelle de Sébastien Rémy, ainsi que quelques comptes rendus d'ouvrages et d'expositions récents.

Jérôme Glicenstein

avril 2016 